\author{
Military Technical College \\ Kobry El-Kobbah, \\ Cairo, Egypt
}

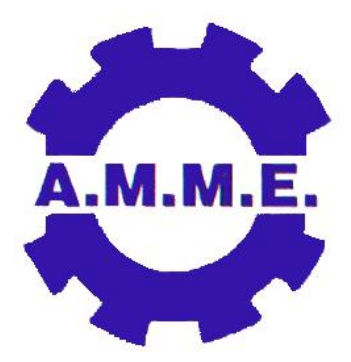

14th International Conference on

Applied Mechanics and

Mechanical Engineering.

\title{
BIODIESEL FROM SEABUCKTHORN OIL
}

By

R.DINESH *

S.S.VARUN SHANKAR **

\begin{abstract}
:
The world is confronted with the twin crises of fossil fuel depletion and environmental degradation. Hence it is necessary to look for alternative fuels, which can be produced from materials available within the country. Although vegetable oils can be fuel for diesel engines, their high viscosities, low volatilities and poor cold flow properties have led to the investigation of their various derivatives. Among the different possible sources, fatty acid methyl esters, known as Biodiesel fuel derived from triglycerides (vegetable oil and animal fates) by transesterification with methanol, present the promising alternative substitute to diesel fuels and have received the most attention now a days. The main advantages of using Biodiesel are its renewability, better quality exhaust gas emission, its biodegradability and the organic carbon present in it is photosynthetic in origin. It does not contribute to a rise in the level of carbon dioxide in the atmosphere and consequently to the green house effect. Here we use sea-buckthorns (Hippophae L). Oils from seabuckthorn seeds and pulp differ considerably in fatty acid composition. While linoleic acid and $\alpha$-linolenic acid are the major fatty acids in seed oil, sea buckthorn pulp oil contains approximately $65 \%$ combination of the monounsaturated fatty acid, palmitoleic acid, saturated fatty acid and palmitic acid. It has low viscosity among vegetable oils. Due to low acidic value we use base catalyst, activated calcium oxide.
\end{abstract}

\section{Keywords:}

seabuckthorn, hippophae L, low viscosity, activated calcium oxide 
* Velammal Engg College, Chennai-66

\section{Introduction:}

Multiple impacts occur in rigid bodies when several collisions take place at multiple contact points simultaneously. Such problems pose many difficulties and unanswered questions [1-3]. This problem was studied by many researchers and several models were introduced. Only few models produce unique and energetically consistent solutions [4-7]. Recently, Ceanga and Hurmuzlu [5] introduced a method that produced unique and energetically consistent solutions in frictionless multiple impact problems. They developed a new approach that used the energetic coefficient of restitution, and proposed a new constant, that was called the "Impulse Correlation Ratio" (ICR). This parameter is physically meaningful and more effective in dealing with multiple impact problems. They applied the method to the multiple impact problems in a linear $\mathrm{N}$-ball chain.

The rocking block problem, where multiple impacts are likely to occur, is one of the simplest rigid body impact problems that can involve multiple collisions. Understanding the physics of a rocking block is also important in applications such as robotics, buildings and tall structures subject to earthquakes, and motion of water tanks. Housner [8] introduced the first study to derive the mathematical equations of a free-standing rigid block under base excitation. This model was called the simple rocking model (SRM); where plastic impact at the collision point is assumed (1-DOF). The block equations of motion was described by piecewise nonlinear equations depending on the sign of the rotation angle. Many researchers analyzed the block response due to different earthquake inputs using Housner's approach [9-14].

The objective of this study is to extend the method given in Ceanga and Hurmuzlu [5] to solve the rocking block problem. Here, we consider a rigid block with two rocking ends on smooth surfaces that are set at arbitrary angles. Then, we develop a solution method based on the rigid body approach and impulse momentum methods. Finally, we verify the approach by conducting a set of experiments, and comparing the theoretical outcomes with the experimental ones.

\section{The Impulse Momentum Method:}

In this section, we summarize the Impulse Momentum Model introduced by Ceanga and Hurmuzlu [5]. Consider the three balls with masses $m_{i}(i=1,2,3)$ that are depicted in Fig. (1). Ball $B_{1}$ strikes the other two balls (with initial velocities of $v_{2}{ }^{-}$and $v_{3}{ }^{-}$) that are in contact at time $\mathrm{t}=\mathrm{t}^{-}$with a velocity of $\mathrm{v}_{1}{ }^{-}$subject to $\mathrm{v}_{1}{ }^{-}>\mathrm{v}_{2}{ }^{-}>\mathrm{v}_{3}{ }^{-}$. The collision causes the two normal impulses $T_{1,2}$ and $T_{2,3}$ as shown in the figure. The problem at hand is to determine the post impact velocities $v_{i}^{+}$. One can write the conservation of linear momentum equations for the three balls, this yields:

$$
\begin{gathered}
m_{1} \Delta v_{1}=-\Delta \tau_{1,2} \\
m_{2} \Delta v_{2}=\Delta \tau_{1,2}-\Delta \tau_{2,3} \\
m_{3} \Delta v_{3}=\Delta \tau_{2,3}
\end{gathered}
$$




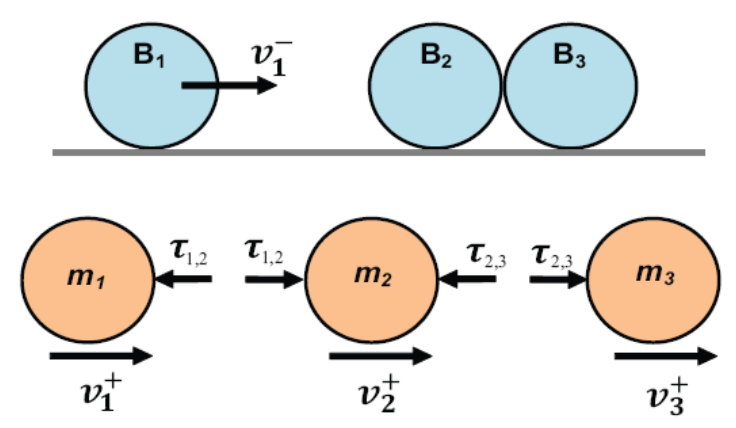

Figure (1): Three ball impact

where, $\Delta \mathrm{v}_{\mathrm{i}}(\mathrm{i}=1 ; 2 ; 3)$ are the changes in velocities and $\Delta \mathrm{T}_{\mathrm{j}, \mathrm{j}+1}(\mathrm{j}=1,2)$ are the changes in impulses as a result of the collision. Here, we have three equations in terms of the three post impact velocities and the two changes that occur in normal impulses. Using the hypothesis from Ceanga and Hurmuzlu [5] to establish a relationship between the normal impulses when $B_{2}$ establishes contact and initiates a collision with $B_{3}$ during the impact of $B_{1}$ and $B_{2}$. This relationship is given by:

$$
\Delta \tau_{2,3}=\alpha \Delta \tau_{1,2}
$$

where, $\alpha$ is called the impulse correlation ratio (ICR) and $0<\alpha<1$. Accordingly, we can get:

$$
\begin{gathered}
v_{1}=v_{1}^{-}-\frac{1}{m_{1}} \tau_{1,2} \\
v_{2}=v_{2}^{-}+\frac{1}{m_{2}}(1-\alpha) \tau_{1,2} \\
v_{3}=v_{3}^{-}+\frac{1}{m_{3}} \tau_{2,3}
\end{gathered}
$$

Next, we compute the work done during the compression $\left(\mathrm{W}_{\mathrm{c}}\right)$ and restitution $\left(\mathrm{W}_{\mathrm{r}}\right)$ phases and use the definition of Stronge's energetic coefficient of restitution [4]:

$$
e_{i, j}^{2}=\frac{W r_{j, j+1}}{W c_{j, j+1}}=\frac{\int_{\tau_{j, j+1}^{c}}^{\tau_{j, j+1}^{f}}\left(v_{j}-v_{j+1}\right) d \tau_{j, j+1}}{\int_{0}^{\tau_{j, j+1}^{c}}\left(v_{j}-v_{j+1}\right) d \tau_{j, j+1}}
$$

where, $e_{j, j+1}$ is the coefficient of restitution between $B_{j}$ and $B_{j+1}(j=1,2)$. The superscripts " $c$ " and " $\mathrm{f}$ " refer to the maximum compression and final values respectively. Thus we can obtain the following equations: 


$$
\begin{aligned}
& e_{1,2}^{2} \int_{0}^{\tau_{1,2}^{c}}\left(v_{1}-v_{2}\right) d \tau_{1,2}+\int_{\tau_{1,2}^{c}}^{\tau_{1,2}^{f}}\left(v_{1}-v_{2}\right) d \tau_{1,2}=0 \\
& e_{2,3}^{2} \int_{0}^{\tau_{2,3}^{c}}\left(v_{2}-v_{3}\right) d \tau_{2,3}+\int_{\tau_{2,3}^{c}}^{\tau_{2,3}^{f}}\left(v_{2}-v_{3}\right) d \tau_{2,3}=0
\end{aligned}
$$

The maximum compression impulses $\mathrm{T}_{\mathrm{j}, \mathrm{j}+1}^{\mathrm{c}}$ can be obtained by setting $\mathrm{V}_{\mathrm{j}+1}=\mathrm{V}_{\mathrm{j}}$ and solving for them. Hence, we get:

$$
\begin{aligned}
& \tau_{1,2}^{c}=\left(v_{1}^{-}-v_{2}^{-}\right)\left(v_{2}^{-}-v_{3}^{-}\right) m_{1} m_{2} / \lambda_{2} \\
& \tau_{2,3}^{c}=\left(\lambda_{1}+\lambda_{2}\right)\left(\lambda_{3} / \lambda_{2}\right)
\end{aligned}
$$

Finally, the post impact velocities can now be computed as follows

$$
\begin{gathered}
v_{1}^{+}=v_{1}^{-}-\frac{1}{m_{1}} \tau_{1,2}^{f} \\
v_{2}^{+}=v_{2}^{*}-\frac{1}{m_{2}}\left(\tau_{2,3}^{f}-\alpha \tau_{1,2}^{f}\right) \\
v_{3}^{+}=v_{3}^{-}+\frac{1}{m_{3}} \tau_{2,3}^{f}
\end{gathered}
$$

where

$$
\begin{gathered}
\tau_{1,2}^{f}=\left(\lambda_{1} / \lambda_{2}\right)\left(v_{2}^{-}-v_{3}^{-}\right) m_{2} \\
v_{2}^{*}=v_{2}^{-}+\left(\lambda_{1} / \lambda_{2}\right)\left(v_{2}^{-}-v_{3}^{-}\right)(1-\alpha) \\
\tau_{2,3}^{f}=\left(\lambda_{1}+\lambda_{2}\right)\left(\lambda_{3} / \lambda_{2}\right) \lambda_{4}
\end{gathered}
$$

with

$$
\begin{aligned}
& \lambda_{1}=\left(v_{1}^{-}-v_{2}^{-}\right)\left(1+e_{1,2}\right) m_{1} \\
& \lambda_{2}=\left(v_{2}^{-}-v_{3}^{-}\right)\left[(1-\alpha) m_{1}+m_{2}\right] \\
& \lambda_{3}=\left(v_{2}^{-}-v_{3}^{-}\right) m_{2} m_{3} /\left(m_{2}+m_{3}\right) \\
& \lambda_{4}=1+e_{2,3}\left[1-\alpha\left(m_{2} / m_{3}+1\right)\left(1+\lambda_{2} / \lambda_{1}\right)^{-2}\right]^{1 / 2}
\end{aligned}
$$

\section{The Rocking Block Problem:}

Consider the system shown in Fig. (2). The impact problem takes place as a result of the block striking the left surface $\left(\right.$ at $\mathrm{O}_{1}$ ) while resting on the right surface $\left(\right.$ at $\left.\mathrm{O}_{2}\right)$. The block is symmetrical with a width of $2 \mathrm{~b}$, a height of $2 \mathrm{~h}$, mass $\mathrm{m}$, and a centroidal moment of inertia of $I_{\mathrm{cm}}$. To simplify the calculations, we choose an inertial coordinate system whose origin coincides with the center of the block and its $x$ and $y$ axes are parallel to the respective edges of the block at the impact instance (i.e. the block is always horizontal at the moment of impact). At the instant immediately before impact, the block is undergoing a noncentroidal rotation about $\mathrm{O}_{2}$ (where the initial velocity at $\mathrm{O}_{2}$ is equal to zero) with an angular velocity of $\omega$. In addition, we consider frictionless contacts only and choose 
surface inclinations of $\theta_{1}$ and $\theta_{2}$ at the respective contact points $O_{1}$ and $O_{2}$. The objective of solving the impact problem is to compute the angular velocity of the block and the linear velocity of its mass center immediately after impact in terms of the pre-impact velocities. The problem can be cast in terms of five scalar unknowns for the frictionless case: three post impact velocities and the magnitude of the normal impulses $\mathrm{T}_{1}{ }^{\mathrm{n}}$, and $\mathrm{T}_{2}{ }^{\mathrm{n}}$ ) at the two contact points. Here, since we consider the frictionless case, we will ignore the superscripts " $n$ " in the remainder of the paper and denote the impulses at contact points as $T_{1}$, and $T_{2}$. The solution is not straightforward. One can obtain three equations from the conservation of linear and angular momenta. An additional equation can be obtained from the application of the concept of coefficient of restitution at the point of collision $\left(\mathrm{O}_{1}\right)$. Yet, one encounters difficulty in obtaining a fifth equation. A restitution equation can not be written for the second contact point, because the pre-impact velocity of the block is zero at this point. Thus, the number of equations fall one short of the number of unknowns.

In the next section, we apply the Impulse Correlation Ratio (ICR) concept that was developed in Ceanga and Hurmuzlu [5] to resolve the difficulty that is encountered in the present problem.

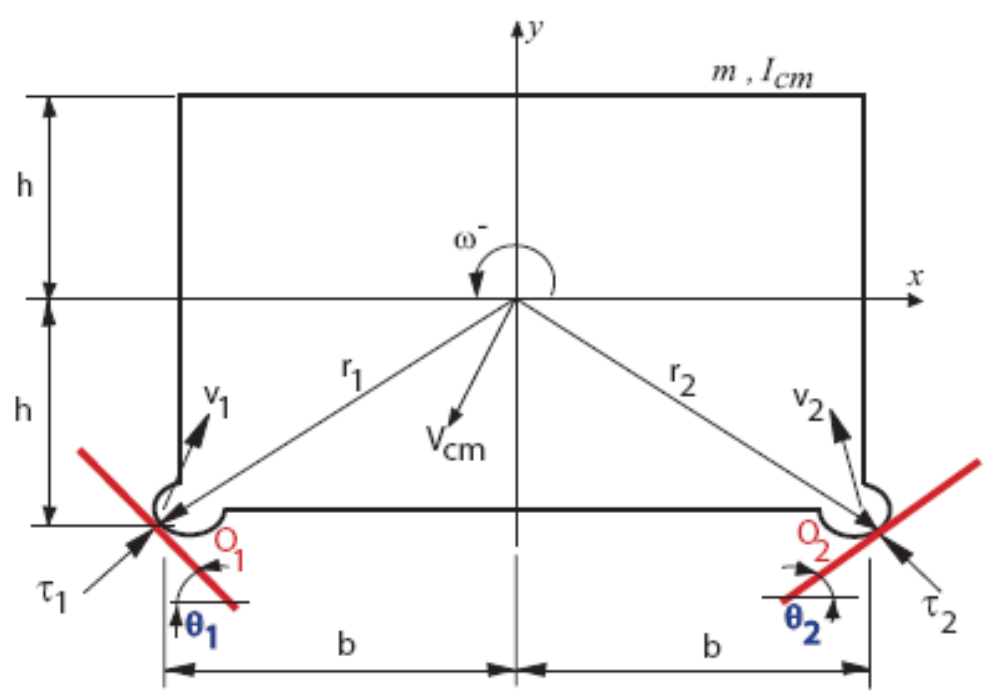

Figure (2): Rigid block diagram at the moment of impact

\section{Velocity-Impulse Relationships:}

Using the law of impulse and momentum, we obtain the following equations:

$$
\begin{gathered}
m \Delta \mathbf{V}_{\mathrm{cm}}=\Delta \tau_{1}+\Delta \tau_{2} \\
I_{c m} \Delta \omega=-\left(\Delta \tau_{1} \times \mathbf{r}_{1}-\Delta \tau_{2} \times \mathbf{r}_{2}\right)
\end{gathered}
$$

where, $\Delta V_{c m}$ and $\Delta \omega$ are the changes in the linear and angular velocity vectors of the mass center and the block respectively, and $r_{1}$ and $r_{2}$ are the vectors from the mass center to the contact points. The kinematic relationship among the velocities at the contact points and the mass center can be written as follows: 


$$
\begin{aligned}
& \mathrm{v}_{1}=\mathrm{V}_{\mathrm{cm}}+\omega \times \mathrm{r}_{1} \\
& \mathrm{v}_{\mathbf{2}}=\mathrm{V}_{\mathrm{cm}}+\omega \times \mathrm{r}_{2}
\end{aligned}
$$

where $v_{1}$ and $v_{2}$ are the velocities of the block at $O_{1}$ and $O_{2}$ respectively (see Fig. (2)). For the remainder of the paper, we remove the vector notation. Since, we have no friction, we will use $\Delta \mathrm{T}_{\mathrm{i}}$ and $\Delta \mathrm{V}_{\mathrm{i}}(\mathrm{i}=1,2)$ for the changes in magnitudes of the normal components of the respective impulses and velocities. In addition, we use $\Delta v_{i}{ }^{x}$ and $\Delta v_{i}{ }^{y} \quad(i=1,2)$ for the changes in the magnitudes of the velocity vectors in the $x$ and $y$ directions respectively.

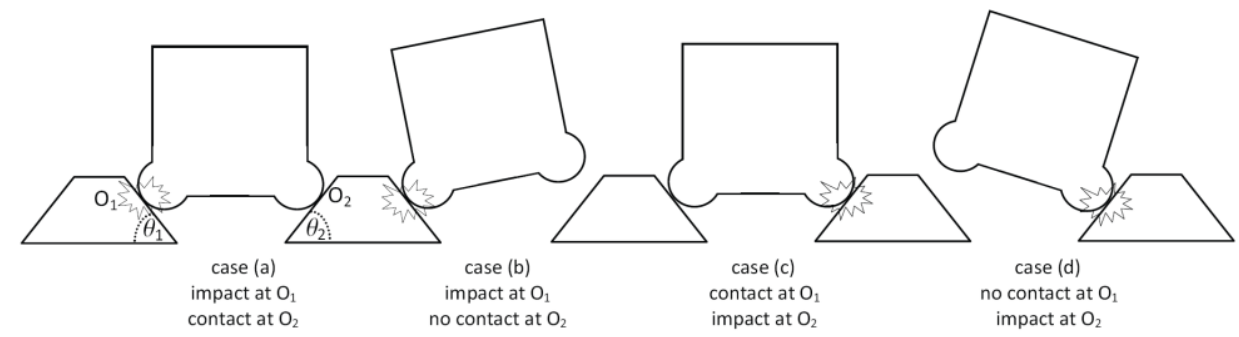

Figure (3): Rigid block contact-impact cases

Now, use Eqs. (20), (21), (22), and (23) to derive the following velocity expressions in terms of the impulses at the contact points:

$$
\begin{gathered}
\Delta v_{1}^{x}=\Delta v_{2}^{x}=\frac{b}{\Gamma_{1}}\left[\Gamma_{3}\left(\Delta \tau_{2} \cos \theta_{2}-\Delta \tau_{1} \cos \theta_{1}\right)+\Gamma_{4}\left(\Delta \tau_{1} \sin \theta_{1}-\Delta \tau_{2} \sin \theta_{2}\right)\right] \\
\Delta v_{1}^{y}=\frac{b}{\Gamma_{1}}\left[\Gamma_{3}\left(\Delta \tau_{2} \sin \theta_{2}-\Delta \tau_{1} \sin \theta_{1}\right)+\Gamma_{2} \Delta \tau_{1} \cos \theta_{1}+\Gamma_{5} \Delta \tau_{2} \cos \theta_{2}\right] \\
\Delta v_{2}^{y}=\frac{b}{\Gamma_{1}}\left[-\Gamma_{3}\left(\Delta \tau_{2} \sin \theta_{2}-\Delta \tau_{1} \sin \theta_{1}\right)+\Gamma_{2} \Delta \tau_{2} \cos \theta_{2}+\Gamma_{5} \Delta \tau_{1} \cos \theta_{1}\right]
\end{gathered}
$$

where

$$
\begin{aligned}
& \Gamma_{1}=b I_{c m} m \\
& \Gamma_{2}=I_{c m}+b^{2} m \\
& \Gamma_{3}=h b m \\
& \Gamma_{4}=I_{c m}+m h^{2} \\
& \Gamma_{5}=I_{c m}-b^{2} m
\end{aligned}
$$

In this rigid block example, there are four possible cases that may arise during various phases of the collision process. Figure. (3) Shows these four cases. Note that, in Fig. (3) although the block is horizontal, the gap at the non-contacting end is exaggerated for illustrative purposes. In addition, "contact" at a particular end means that the gap at that end is closed with zero initial normal relative velocity.

\subsection{Case (a) Impact at 01 - Contact at O2:}


Substituting $\Delta \mathrm{T}_{2}=\alpha \Delta \mathrm{T}_{1}$ into Eqs. (24), (25), and (26), we obtain the following equations:

$$
\begin{gathered}
\Delta v_{1}^{x}=\Delta v_{2}^{x}=\frac{\Delta \tau_{1} b}{\Gamma_{1}}\left[\Gamma_{3}\left(\alpha \cos \theta_{2}-\cos \theta_{1}\right)+\Gamma_{4}\left(\sin \theta_{1}-\alpha \sin \theta_{2}\right)\right] \\
\Delta v_{1}^{y}=\frac{\Delta \tau_{1} b}{\Gamma_{1}}\left[\Gamma_{3}\left(\alpha \sin \theta_{2}-\sin \theta_{1}\right)+\Gamma_{2} \cos \theta_{1}+\Gamma_{5} \alpha \cos \theta_{2}\right] \\
\Delta v_{2}^{y}=\frac{\Delta \tau_{1} b}{\Gamma_{1}}\left[\Gamma_{3}\left(\sin \theta_{1}-\alpha \sin \theta_{2}\right)+\Gamma_{2} \alpha \cos \theta_{2}+\Gamma_{5} \cos \theta_{1}\right]
\end{gathered}
$$

\subsection{Case (b) Impact at 01 - No Contact at O2:}

Substituting $\Delta \mathrm{T}_{2}=0$ into Eqs. (24), (25), and (26), we obtain the following equations:

$$
\begin{gathered}
\Delta v_{1}^{x}=\Delta v_{2}^{x}=\frac{\Delta \tau_{1} b}{\Gamma_{1}}\left[-\Gamma_{3} \cos \theta_{1}+\Gamma_{4} \sin \theta_{1}\right] \\
\Delta v_{1}^{y}=\frac{\Delta \tau_{1} b}{\Gamma_{1}}\left[-\Gamma_{3} \sin \theta_{1}+\Gamma_{2} \cos \theta_{1}\right] \\
\Delta v_{2}^{y}=\frac{\Delta \tau_{1} b}{\Gamma_{1}}\left[\Gamma_{3} \sin \theta_{1}+\Gamma_{5} \cos \theta_{1}\right]
\end{gathered}
$$

\subsection{Case (c) Contact at 01 - Impact at O2:}

Substituting $\Delta \mathrm{T}_{1}=\alpha \Delta \mathrm{T}_{2}$ into Eqs. (24), (25), and (26), we obtain the following equations:

$$
\begin{gathered}
\Delta v_{1}^{x}=\Delta v_{2}^{x}=\frac{\Delta \tau_{2} b}{\Gamma_{1}}\left[\Gamma_{3}\left(\cos \theta_{2}-\alpha \cos \theta_{1}\right)+\Gamma_{4}\left(\alpha \sin \theta_{1}-\sin \theta_{2}\right)\right] \\
\Delta v_{1}^{y}=\frac{\Delta \tau_{2} b}{\Gamma_{1}}\left[\Gamma_{3}\left(\sin \theta_{2}-\alpha \sin \theta_{1}\right)+\Gamma_{2} \alpha \cos \theta_{1}+\Gamma_{5} \cos \theta_{2}\right] \\
\Delta v_{2}^{y}=\frac{\Delta \tau_{2} b}{\Gamma_{1}}\left[\Gamma_{3}\left(\alpha \sin \theta_{1}-\sin \theta_{2}\right)+\Gamma_{2} \cos \theta_{2}+\Gamma_{5} \alpha \cos \theta_{1}\right]
\end{gathered}
$$

\subsection{Case (d) No Contact at 01 - Impact at O2:}

Substituting $\Delta \mathrm{T}_{1}=0$ into Eqs. (24), (25), and (26), we obtain the following equations:

$$
\begin{gathered}
\Delta v_{1}^{x}=\Delta v_{2}^{x}=\frac{\Delta \tau_{2} b}{\Gamma_{1}}\left[\Gamma_{3} \cos \theta_{2}+\Gamma_{4} \sin \theta_{2}\right] \\
\Delta v_{1}^{y}=\frac{\Delta \tau_{2} b}{\Gamma_{1}}\left[\Gamma_{3} \sin \theta_{2}+\Gamma_{5} \cos \theta_{2}\right] \\
\Delta v_{2}^{y}=\frac{\Delta \tau_{2} b}{\Gamma_{1}}\left[-\Gamma_{3} \sin \theta_{2}+\Gamma_{2} \cos \theta_{2}\right]
\end{gathered}
$$

For all cases $a, b, c$, and $d$ we compute the changes in normal velocities of both ends as 
follows:

$$
\begin{gathered}
\Delta v_{1}=\Delta v_{1}^{x} \sin \theta_{1}+\Delta v_{1}^{y} \cos \theta_{1} \\
\Delta v_{2}=-\Delta v_{2}^{x} \sin \theta_{2}+\Delta v_{2}^{y} \cos \theta_{2}
\end{gathered}
$$

\section{Solution Procedure:}

In this section we present the solution to the block multiple impact problems that are considered in this paper. We use the equations presented in the previous section to obtain a piecewise solution of the impact problem. At the onset of the collision, the block strikes the external surface at $O_{1}$ while resting at $O_{2}$ (this is case (a); where $v_{1}^{-} \neq 0$ and $v_{2}^{-} \neq 0$, or more specifically $\omega^{-}=\omega_{0} \neq 0$ ). There are two possible bouncing patterns that result from the collision at $\mathrm{O}_{1}$, single impact and simultaneous collision.

\subsection{Single Impact:}

This case arises when the non-impacting end bounces at the onset of the collision (i.e. its normal velocity becomes positive immediately). This means that the slope of the normal velocity $v_{2}$ is positive at the onset of impact (case (b)). Thus, using Eqs. (31) and (33) we may write the condition for the occurrence of this case as follows:

$$
\begin{gathered}
\frac{d v_{2}}{d \tau_{1}}=-\frac{d v_{2}^{x}}{d \tau_{1}} \sin \theta_{2}+\frac{d v_{2}^{y}}{d \tau_{1}} \cos \theta_{2}= \\
\frac{\left(h^{2}+b^{2}\right) \cos \left(\theta_{1}+\theta_{2}\right)+\left[-2 \frac{I_{c m}}{m}+\left(h^{2}-b^{2}\right)\right] \cos \left(\theta_{1}-\theta_{2}\right)-2 h b \sin \left(\theta_{1}-\theta_{2}\right)}{2 \frac{I_{c m}}{m}+\left(h^{2}+b^{2}\right)+\left(h^{2}-b^{2}\right) \cos 2 \theta_{2}+2 h b \sin 2 \theta_{2}}>0
\end{gathered}
$$

The maximum compression impulse at $\mathrm{O}_{1}, \mathrm{~T}_{1}{ }^{\mathrm{C}}$, can now be found by setting $\mathrm{v}_{1}=0$ in Eq. (40). Then, we obtain:

$$
\tau_{1}^{c}=\frac{2 \Gamma_{1} \omega_{0} \cos \theta_{1}}{\Gamma_{2} \cos ^{2} \theta_{1}+\sin \theta_{1}\left(-2 \Gamma_{3} \cos \theta_{1}+\Gamma_{4} \sin \theta_{1}\right)}
$$

The impulse at the end of the collision at $\mathrm{O}_{1}, \mathrm{~T}_{1}{ }^{\dagger}$, can be found by using the energetic definition of the coefficient of restitution [4]:

$$
e_{1}^{2} \int_{0}^{\tau_{1}^{c}} \Delta v_{1} d \tau_{1}+\int_{\tau_{1}^{c}}^{\tau_{1}^{f}} \Delta v_{1} d \tau_{1}=0
$$

where $e_{1}$ is the coefficient of restitution at $\mathrm{O}_{1}$. By solving this equation, we obtain: 


$$
\tau_{1}^{f}=\frac{2\left(1+e_{1}\right) \Gamma_{1} \omega_{0} \cos \theta_{1}}{\Gamma_{2} \cos ^{2} \theta_{1}+\sin \theta_{1}\left(-2 \Gamma_{3} \cos \theta_{1}+\Gamma_{4} \sin \theta_{1}\right)}
$$

The post-impact velocities can be found by substituting the final impulse into the respective velocity expressions. So, the final velocities in this case are:

$$
\begin{gathered}
v_{1}^{x}=v_{2}^{x}=\frac{2\left(1+e_{1}\right) b \omega_{0} \cos \theta_{1}\left(-\Gamma_{3} \cos \theta_{1}+\Gamma_{4} \sin \theta_{1}\right)}{\Gamma_{6}} \\
v_{1}^{y}=-2 b \omega_{0}\left[1-\frac{\left(1+e_{1}\right) \cos \theta_{1}\left(\Gamma_{2} \cos \theta_{1}-\Gamma_{3} \sin \theta_{1}\right)}{\Gamma_{6}}\right] \\
v_{2}^{y}=\frac{2\left(1+e_{1}\right) b \omega_{0} \cos \theta_{1}\left(\Gamma_{5} \cos \theta_{1}+\Gamma_{3} \sin \theta_{1}\right)}{\Gamma_{6}}
\end{gathered}
$$

where,

$$
\Gamma_{6}=\Gamma_{2} \cos ^{2} \theta_{1}+\sin \theta_{1}\left(-2 \Gamma_{3} \cos \theta_{1}+\Gamma_{4} \sin \theta_{1}\right)
$$

\subsection{Simultaneous Collision At Both Ends:}

If the condition in Eq. (42) is violated, simultaneous impacts at $\mathrm{O}_{1}$ and $\mathrm{O}_{2}$ take place. Initially, we have a case where there is an impact at $\mathrm{O}_{1}$ and contact at $\mathrm{O}_{2}$ (case (a)). Using Eqs. (28), (29), and (40) and setting $v_{1}=0$ yields the maximum compression impulse for the first impact at $\mathrm{O}_{1}$ as follows:

$$
\tau_{1}^{c}=\frac{2 b \omega_{0} \cos \theta_{1}}{\Gamma_{7} \cos \theta_{1}+\Gamma_{8} \sin \theta_{1}}
$$

where,

$$
\begin{aligned}
& \Gamma_{7}=\frac{b}{\Gamma_{1}}\left[\Gamma_{3}\left(\alpha \sin \theta_{2}-\sin \theta_{1}\right)+\Gamma_{2} \cos \theta_{1}+\Gamma_{5} \alpha \cos \theta_{2}\right] \\
& \Gamma_{8}=\frac{b}{\Gamma_{1}}\left[\Gamma_{3}\left(\alpha \cos \theta_{2}-\cos \theta_{1}\right)+\Gamma_{4}\left(\sin \theta_{1}-\alpha \sin \theta_{2}\right)\right]
\end{aligned}
$$

We use the energetic definition of the coefficient of restitution to write:

$$
e_{1}^{2} \int_{0}^{\tau_{1}^{c}} \Delta v_{1} d \tau_{1}+\int_{\tau_{1}^{c}}^{\tau_{1}^{f}} \Delta v_{1} d \tau_{1}=0
$$

The final impulse for the first collision at $O_{1}$ can be found by substituting the velocity expressions in Eq. (53) and solving for $\mathrm{T}_{1}{ }^{\mathrm{f}}$, which yields: 


$$
\tau_{1}^{f}=\frac{2\left(1+e_{1}\right) \Gamma_{1} \omega_{0} \cos \theta_{1}}{\Gamma_{9}+\Gamma_{10}}
$$

where,

$$
\begin{aligned}
\Gamma_{9} & =\Gamma_{2} \cos ^{2} \theta_{1}+\sin \theta_{1}\left[\Gamma_{3} \alpha \cos \theta_{2}+\Gamma_{4}\left(\sin \theta_{1}-\alpha \sin \theta_{2}\right)\right] \\
\Gamma_{10} & =\cos \theta_{1}\left[\Gamma_{5} \alpha \cos \theta_{2}+\Gamma_{3}\left(-2 \sin \theta_{1}+\alpha \sin \theta_{2}\right)\right]
\end{aligned}
$$

Now, the velocity expressions at the end of the $\mathrm{O}_{1}$ collision can be obtained by substituting $\mathrm{T}_{1}{ }^{\mathrm{f}}$ in the respective velocity equations to yield:

$$
\begin{gathered}
v_{1}^{x}=v_{2}^{x *}=\frac{2\left(1+e_{1}\right) \Gamma_{1} \Gamma_{8} \omega_{0} \cos \theta_{1}}{\Gamma_{9}+\Gamma_{10}} \\
v_{1}^{y *}=2 b \omega_{0}\left[-1+\frac{\left(1+e_{1}\right) I_{c m} m \Gamma_{7} \cos \theta_{1}}{\Gamma_{9}+\Gamma_{10}}\right] \\
v_{2}^{y *}=\frac{2\left(1+e_{1}\right) \Gamma_{1} \Gamma_{11} \omega_{0} \cos \theta_{1}}{\Gamma_{9}+\Gamma_{10}}
\end{gathered}
$$

where,

$$
\Gamma_{11}=\frac{b}{\Gamma_{1}}\left[\Gamma_{3}\left(\sin \theta_{1}-\alpha \sin \theta_{2}\right)+\Gamma_{2} \alpha \cos \theta_{2}+\Gamma_{5} \cos \theta_{1}\right]
$$

Although the impact at $\mathrm{O}_{1}$ ends, the collision at $\mathrm{O}_{2}$ continues. The impulse at $\mathrm{O}_{2}$ when the collision at $\mathrm{O}_{1}$ ends can be written as :

$$
\tau_{2}^{*}=\alpha \tau_{1}^{f}=\frac{2\left(1+e_{1}\right) \Gamma_{1} \alpha \omega_{0} \cos \theta_{1}}{\Gamma_{9}+\Gamma_{10}}
$$

Now that we have impact only at $\mathrm{O}_{2}$, we consider the case where there is an impact at $\mathrm{O}_{2}$ and no contact at $\mathrm{O}_{1}$ (case $(\mathrm{d})$ ). The velocities during this interval can be written as follows :

$$
\begin{gathered}
v_{1}^{x}=v_{2}^{x}=v_{1}^{x *}+\frac{\left(\tau_{2}-\tau_{2}^{*}\right) b\left(\Gamma_{3} \cos \theta_{2}-\Gamma_{4} \sin \theta_{2}\right)}{\Gamma_{1}} \\
v_{1}^{y}=v_{1}^{y *}+\frac{\left(\tau_{2}-\tau_{2}^{*}\right) b\left(\Gamma_{5} \cos \theta_{2}+\Gamma_{3} \sin \theta_{2}\right)}{\Gamma_{1}} \\
v_{2}^{y}=v_{2}^{y *}+\frac{\left(\tau_{2}-\tau_{2}^{*}\right) b\left(\Gamma_{2} \cos \theta_{2}-\Gamma_{3} \sin \theta_{2}\right)}{\Gamma_{1}}
\end{gathered}
$$

We use Eqs. (62), (64), and (41) along with the condition $v_{2}=0$ to obtain the maximum compression impulse for the first collision at $\mathrm{O}_{2}$ as follows: 


$$
\tau_{2}^{c}=\frac{\left(-v_{2}^{y *}+\Gamma_{12} \tau_{2}^{*}\right) \cos \theta_{2}+\left(v_{2}^{x *}-\Gamma_{13} \tau_{2}^{*}\right) \sin \theta_{2}}{\Gamma_{12} \cos \theta_{2}-\Gamma_{13} \sin \theta_{2}}
$$

where,

$$
\begin{aligned}
\Gamma_{12} & =\frac{b\left(\Gamma_{2} \cos \theta_{2}-\Gamma_{3} \sin \theta_{2}\right)}{\Gamma_{1}} \\
\Gamma_{13} & =\frac{b\left(\Gamma_{3} \cos \theta_{2}-\Gamma_{4} \sin \theta_{2}\right)}{\Gamma_{1}}
\end{aligned}
$$

Now, we use the energetic definition of the coefficient of restitution to write:

$$
e_{2}^{2} \int_{0}^{\tau_{2}^{c}} \Delta v_{2} d \tau_{2}+\int_{\tau_{2}^{c}}^{\tau_{2}^{f}} \Delta v_{2} d \tau_{2}=0
$$

We solve this equation to find the final impulse for the first $\mathrm{O}_{2}$ collision, and we obtain:

$$
\tau_{2}^{f}=\frac{\Gamma_{1} \Gamma_{15}}{\Gamma_{17}\left(\Gamma_{2} \cos ^{2} \theta_{2}+\Gamma_{4} \sin ^{2} \theta_{2}-\Gamma_{3} \sin \left(2 \theta_{2}\right)\right)}\left[\Gamma_{14}+e_{2} \sqrt{-\Gamma_{16} \alpha+\Gamma_{14}^{2}}\right]
$$

where,

$$
\begin{gathered}
\Gamma_{14}=\left(-\Gamma_{4}-\Gamma_{5}\right) \cos \left(\theta_{1}+\theta_{2}\right)+m\left[\left(h^{2}+b^{2}\right) \cos \left(\theta_{1}-\theta_{2}\right)-2 h b \sin \left(\theta_{1}+\theta_{2}\right)\right] \\
\Gamma_{15}=\left(1+e_{1}\right) \omega_{0} \cos \theta_{1} \\
\Gamma_{16}=\left[\left(\Gamma_{5}-\Gamma_{4}\right) \cos \left(\theta_{1}-\theta_{2}\right)+\left(\Gamma_{4}+\Gamma_{5}\right) \cos \left(\theta_{1}+\theta_{2}\right)\right. \\
\left.+2 \Gamma_{3} \sin \left(\theta_{1}+\theta_{2}\right)\right]\left[-\Gamma_{2}-\Gamma_{4}-\left(\Gamma_{2}-\Gamma_{4}\right) \cos \left(2 \theta_{2}\right)+2 \Gamma_{3} \sin \left(2 \theta_{2}\right)\right] \\
\Gamma_{17}=\Gamma_{2} \cos ^{2} \theta_{1}+\sin \theta_{1}\left[\alpha \Gamma_{3} \cos \theta_{2}+\Gamma_{4}\left(\sin \theta_{1}-\alpha \sin \theta_{2}\right)\right] \\
+\cos \theta_{1}\left[\alpha \Gamma_{5} \cos \theta_{2}+\Gamma_{3}\left(-2 \sin \theta_{1}+\alpha \sin \theta_{2}\right)\right]
\end{gathered}
$$

Substituting this final impulse in Eqs. (62), (63), and (64), we obtain the velocities for the end of this stage. Once again, we check the normal velocity at $\mathrm{O}_{1}$. If it is positive, there would be no more impacts. Otherwise, additional impacts may emerge. In this case, the computations will continue in a similar manner. We just switch the notations for the two ends of the block, and follow the procedure that we presented above. The process continues until all normal velocities at the contact points become positive. We have developed software routines in Mathematica that carries out this procedure and automatically stops when collisions at both ends cease. Finally, we may obtain the upper limit of the Impulse Correlation Ratio by considering the inside of the square root in Eq. (68) as follows:

$$
\alpha \leq \frac{\Gamma_{14}^{2}}{\Gamma_{16}}
$$




\section{Experimental Study:}

We conducted a set of experiments to verify the methodology that we have presented in the present paper. The experimental set-up was designed such that the angular orientation of one contact surface could be freely adjusted and the second contact surface was fixed in a horizontal orientation. Figure (4.a) depicts a photograph of the experimental setup. Two heavy (much heavier than the mass of the block) rigid steel cylinders were used as contact surfaces. The angular orientation of the left cylinder and the vertical position of the right cylinder (the contact surface with fixed horizontal orientation) in the figure are adjustable. We ensured that the two contact points of the block would have the same altitude at the impact instance. The drop mechanism consisted of a pneumatic cylinder with a Teflon coated plastic attachment (to reduce horizontal motion of the released end of the block) mounted to the end of its piston. Each experiment was initiated by placing one end of the block on the Teflon coating and triggering the cylinder away from the block (see Fig. (4.a)). The experiments were conducted by using a $2.5 \mathrm{~kg}$ steel block that is shown in Fig. (4.b). The two edges of the block were rounded as shown in the figure in order to ensure point contacts. Several markers were placed on the block and the contact surfaces. A high-speed video camera (1000 frames/second) was used to capture and digitize the motion of the markers during each experiment. Then, using the digitized data the pre and post impact velocities of the block were computed.

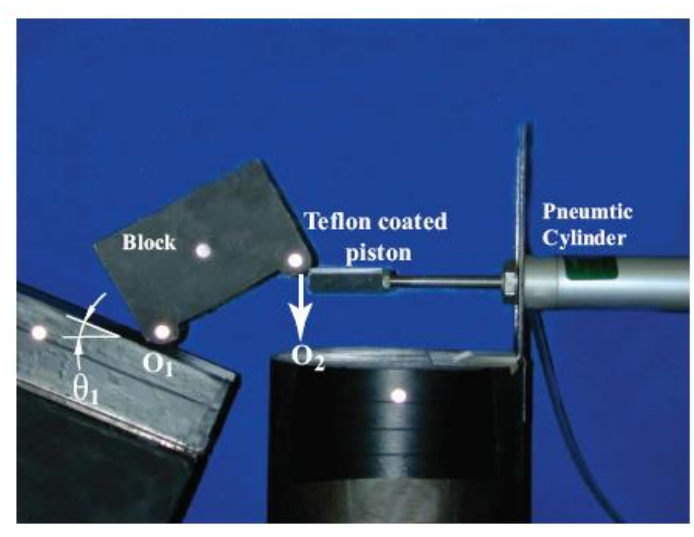

(a)

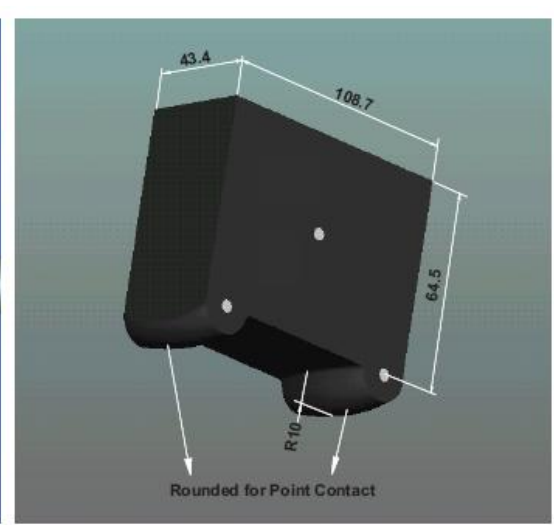

(b)

Figure (4): Experiment; (a)Photo of Experimental Setup, and (b)Geometry of Rigid Block (units are in $\mathrm{mm}$ )

The objective of the experimental study was to verify the theoretical outcomes. For this purpose, we first estimated the kinematic coefficients of restitution at both ends by dropping sample spheres made from the same material as the block on the two cylinders. Then, the ICR was estimated from a single experiment conducted at horizontal contact surfaces $\theta_{1}=\theta_{2}=0^{\circ}$. As a result, the coefficients were estimated as follows: $e_{1}=0.43, e_{2}$ $=0.64$, and $\alpha=0.01$. Subsequently, we have conducted a set of experiments with varying the surface orientations and using the three previously estimated coefficients.

The experiments were performed for $\theta_{1}=\left\{0^{\circ}, 5^{\circ}, 10^{\circ}, 15^{\circ}, 17.5^{\circ}, 20^{\circ}, 22.5^{\circ}\right\}$, and $\theta_{2}=0^{\circ}$. We conducted two sets of experiments. During the first set, the block was resting on the inclined surface at $\mathrm{O}_{1}$ and it impacted the horizontal surface at $\mathrm{O}_{2}$ (the configuration that is shown in Fig. (4.a)). During the second set, the situation was reversed, the block was resting on the horizontal surface at $\mathrm{O}_{2}$ and it struck the inclined surface at $\mathrm{O}_{1}$. For each case, the experiments were repeated three times, releasing the block from various heights 
that varied between 30 and $60 \mathrm{~mm}$. The theoretical and experimental results are depicted in Figs. (5) and (6). Each figure depicts the normalized (with respect to the pre impact vertical velocity) post impact velocities in the normal directions of the contact surfaces. As one can observe from the figures, there is an excellent agreement among the experimental outcomes and the theoretical results. In addition, we observe a clear separation between the velocity slopes at the non-impacting ends. The separation takes place at an angular value of $\theta_{1} \sim 17^{\circ}$. This value was computed analytically as the transition point from single to multiple collisions for the case at hand. In other words, the contacting end of the block separates without impact up to $\theta_{1} \sim 17^{\circ}$. When this angular value exceeded, the contacting end rebounds with interaction with the contact surface. The experimental data clearly exhibit this trend, attesting to the validity of the proposed methodology.

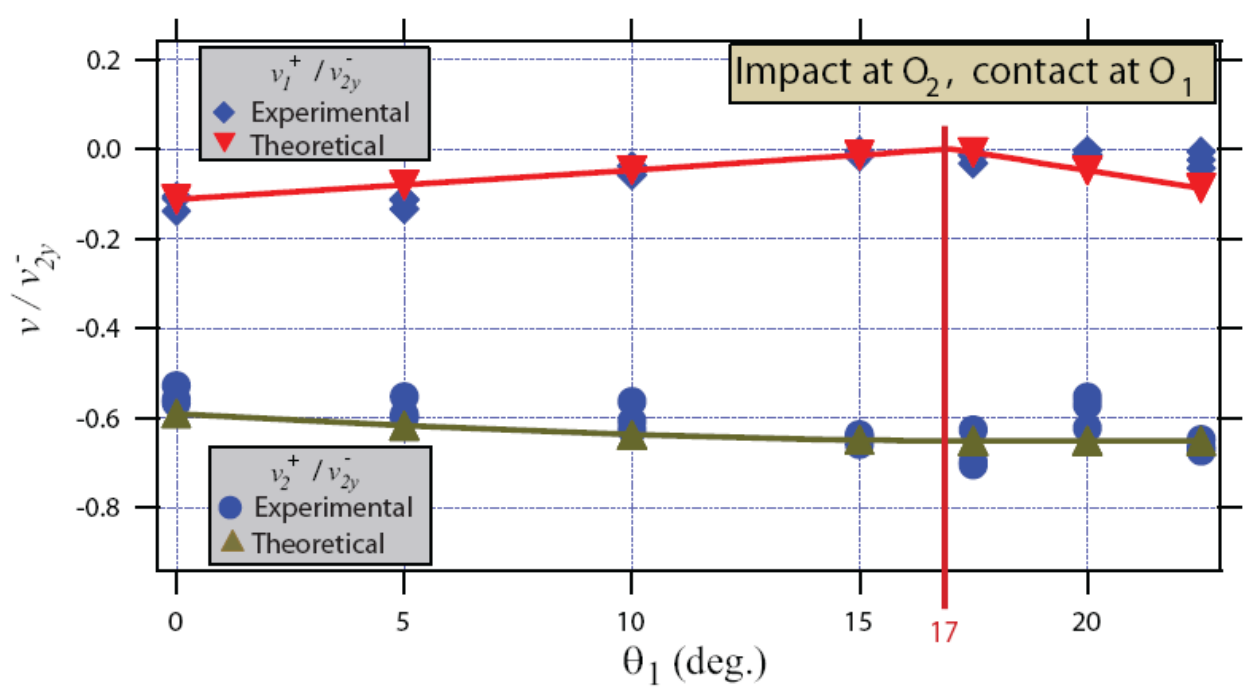

Figure (5): Comparison of experimental and theoretical results, subset \# 1

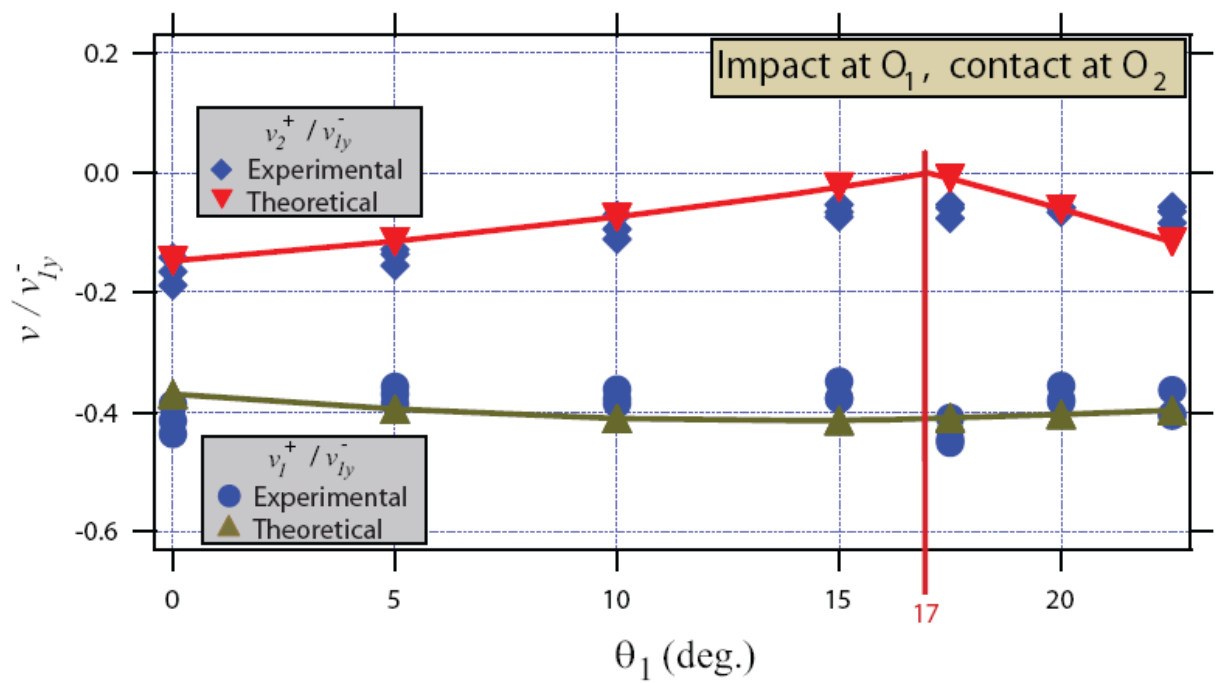

Figure (5): Comparison of experimental and theoretical results, subset \# 2

\section{Conclusion:}

In this paper we developed a new approach to solve the multiple impact problem of a 
rocking block. The methodology is based on the use of impulse momentum methods. The approach uses the Impulse Correlation Ratio (ICR) that was developed previously to solve the multiple impact problems in a linear chain of balls. The method also utilizes the energetic coefficient of restitution and yields energetically consistent solutions. Our formulation works for inclined foundations at both contact points, which was not studied before. Finally, a set of experiments were conducted to demonstrate the validity of the proposed methodology. We have shown that the experimental outcomes agree with the theoretical results. In addition, as far as the separation at the non-contacting end is concerned, the experiments exhibit the same trend that is predicted by the theory. The problem considered in this study was simplified by neglecting friction at the contact points. This was a necessary simplification in this initial stage of the development. Yet, including friction would be a good natural step for future research efforts.

\section{References:}

[1] Marghitu, D. B., and Hurmuzlu, Y., Three dimensional rigid body collisions with multiple contact points, ASME J. Appl. Mech., 62, 725-732, 1995.

[2] Brogliato, B., Nonsmooth Impact Mechanics: Models, Dynamics and Control, New York: Springer, 1996.

[3] Brach, R. M., Mechanical Impact Dynamics: Rigid Body Collisions, New York: John Wiley and Sons, 1991.

[4] Stronge, W. J., Rigid Body Collisions with Friction, Proc. R. Soc. Lond. A, 431, 169$181,1990$.

[5] Ceanga, V. and Hurmuzlu, Y., A new look at an old problem: Newton's cradle, ASME J. Appl. Mech., 68, 575-583, 2001.

[6] Glocker, C., Concepts for modeling impacts without friction. Acta Mech., 168, 1-19, 2004.

[7] Liu, C., Zhao, Z., and Brogliato, B., Frictionless multiple impacts in Multibody systems: Part I. Theoretical framework, Proc. R. Soc. Lond. A, 464, 3193-3211, 2008.

[8] Housner, G. W., The behavior of inverted pendulum structures during earthquakes, Bulletin of Seismological Society of America, 53, No. 2, 403-417, 1963.

[9] Priestley, M. J., Evison, R. J., and Carr, A. J., Seismic response analysis of structures free to rock on their foundations, Bull. of the New Zealand Seis. Soc. for Earthquake Engrg., 11, No. 3, 141-150, 1978.

10] Aslam, M., Godden, W. G. and Scalise, D. T., Earthquake rocking response of rigid bodies, J. Struct. Div., ASCE 106, 377-392, 1980.

11] Yim, C. S., Chopra, A. K., and Penzien, J., Rocking response of rigid blocks to earthquakes, J. Earthquake Engrg. and Struct. Dyn., 8, No. 6, 565-587, 1980.

12] Spanos, P. D., and Koh, A. S., Rocking of rigid blocks during harmonic shaking, J. Engrg. Mech. 110, No., 11, 1627-1642, 1984.

13] Hogan, S. J., On the dynamics of rigid block motion under harmonic forcing, Proc. R. Soc. Lond., A 425, 441-476, 1989.

14] Lipscombe, P. R. and Pellegrino, S., Free Rocking of Prismatic Blocks, J. of Eng. Mech., 119, No. 7, 1387-1410, 1993.

\section{Nomenclature:}

I moment of inertia.

ICR impulse correlation ratio.

KE kinetic energy.

$\mathrm{V}$ velocity. 
W work done.

b block width.

e coefficient of restitution.

h block height.

m mass.

$r$ position vector.

v velocity.

a impulse correlation ratio (ICR).

$\theta \quad$ inclination angle.

T impulse.

$\omega \quad$ angular velocity. 